\title{
Métodos de estudio de pacientes con sangrado uterino posmenopáusico. Recomendaciones prácticas
}

\section{Study methods of patients with postmenopausal uterine bleeding. Practical recommendations}

\section{Pierina Rosales ${ }^{1,2 *}$ y Adriana Doren ${ }^{1,2}$}

${ }^{1}$ Departamento de Obstetricia y Ginecología, Universidad Católica del Maule; ${ }^{2}$ Servicio de Obstetricia y Ginecología, Hospital Regional de Talca. Talca, Chile

\section{Resumen}

El sangrado uterino posmenopáusico se define como aquel sangrado que ocurre después del cese definitivo de la menstruación en la etapa reproductiva de la mujer como consecuencia de la claudicación biológica de los ovarios, o como un sangrado no esperado en mujeres con terapia de reposición hormonal sistémica de la menopausia. Representa el 5\% de las consultas ginecológicas y, si bien su origen suele ser por causas benignas, puede requerir una evaluación minuciosa para descartar patologías malignas del endometrio. El objetivo de este trabajo es establecer un flujograma diagnóstico basado en la evidencia para la evaluación de las pacientes con sangrado uterino posmenopáusico.

Palabras clave: Sangrado uterino posmenopáusico. Cáncer endometrial. Biopsia endometrial. Ecografía transvaginal. Histerosonografía. Histerosocopía.

\section{Abstract}

Postmenopausal uterine bleeding is defined as the bleeding that occurs after the last menstruation due to loss of ovarian function, or a non-scheduled bleeding in patients with hormonal therapy. It represents $5 \%$ of the gynecologic visits, and even though its origin is often benign, it requires a thorough evaluation to discard malignant diseases. The objective of these review is to propose a diagnostic algorithm based on the available evidence for the evaluation of patients with postmenopausal uterine bleeding.

Key words: Postmenopausal uterine bleeding. Endometrial cancer. Endometrial biopsy. Transvaginal ultrasound. Hysterosonography. Hysteroscopy.

\section{Introducción}

El sangrado uterino posmenopáusico (SUPM) se define como el sangrado espontáneo que ocurre posterior al cese permanente de la menstruación (más de 1 año de la última regla $)^{1}$, o bien como un sangrado no esperado en pacientes que usan terapia hormonal de la menopausia $(\mathrm{THM})^{2}$. Ocurre en un $4-11 \%$ de las mujeres que han alcanzado la menopausia, especialmente en los primeros años de su ocurrencia ${ }^{3,4}$.

El $80-90 \%$ de los cánceres de endometrio se manifiestan con SUPM ${ }^{5}$; sin embargo, solo un 6-19 \% de estas mujeres presentan una patología maligna del endometrio, y de ahí la importancia de un adecuado y oportuno estudio ${ }^{6,7}$.

\footnotetext{
Correspondencia: _ Fecha de recepción: 11-07-2020

*Pierina Rosales

E-mail: pierirv@yahoo.com

Disponible en internet: 23-12-2021

Rev Chil Obstet Ginecol. 2021;86(5):492-496

www.rechog.com
} 
La principal causa de SUPM es la atrofia endometrial $(60 \%)$, y en estos casos el sangrado se caracteriza por ser leve, autolimitado y no recurrente. Otras causas comunes son pólipos endometriales $(25 \%)$, terapias hormonales con efecto endometrial (15-25\%), hiperplasia endometrial (5-10\%) y cáncer de endometrio (10\%). Menos del $10 \%$ del resto de los sangrados que ocurren en la posmenopausia obedecen a otras alteraciones no relacionadas con patología endometrial, como cáncer de cuello uterino, trastornos de la coagulación, cervicitis o vaginitis, entre otras ${ }^{8,9}$.

Una causa muy poco frecuente de SUPM es la observada en presencia de miomas uterinos, que no producen sangrado uterino en la posmenopausia, y obliga a considerar el diagnóstico de sarcoma uterino. Estos casos deben ser derivados y manejados en conjunto con el equipo de oncología-ginecológica ${ }^{10}$.

Uno de los principales objetivos de la evaluación del SUPM es descartar un cáncer de endometrio, y para ello existen diferentes métodos de evaluación, como son la ecografía transvaginal, la histerosonografía y la histeroscopía. Estas técnicas permiten considerar el realizar una biopsia endometrial. El manejo o tratamiento del SUPM dependerá de la causa encontrada.

\section{Evaluación del sangrado uterino posmenopáusico}

Las mujeres posmenopáusicas que presenten sangrado uterino deben ser evaluadas para descartar patología maligna del endometrio.

La primera parte de la evaluación de la paciente debe incluir siempre un buen interrogatorio y una adecuada exploración física. El sangrado anormal observado en el área genital generalmente se atribuye a un origen intrauterino, pero deben considerarse otras fuentes, como el cuello uterino, la vagina o la vulva, así como también sitios no ginecológicos, como la uretra, la vejiga, el ano-recto y el periné .

La anamnesis debe incluir la descripción detallada de las características del sangrado (frecuencia, duración y volumen de pérdida de sangre), los factores precipitantes (como trauma o coito) y los síntomas asociados (dolor, fiebre, cambios en la función intestinal o miccional). Es importante interrogar sobre comorbilidad, como por ejemplo antecedente de síndrome de ovario poliquístico o anovulación crónica, diabetes mellitus, obesidad, uso de medicamentos como tamoxifeno, anticoagulantes, THM o suplementos herbales que contengan isoflavonas. La historia familiar de cáncer de mama, colon, endometrio u ovario se considera factor de riesgo de patología maligna endometrial.

La exploración física incluye una completa evaluación de la anatomía externa e interna del tracto genital femenino, así como también de las áreas contiguas no ginecológicas, para precisar el origen del sangrado.

Las mujeres bajo THM, en especial aquellas que reciben un estrógeno con una progestina, el sangrado es observable y su patrón de sangrado esperado dependerá del esquema usado'.

En las mujeres que usan THM combinada cíclica, un estrógeno oral o estradiol transdérmico en forma continua, agregándose un progestágeno o progesterona micronizada durante 10-14 días al mes, se espera sangrado mensual en un $80-90 \%$ de los casos. Este sangrado suele ir disminuyendo con los años de uso ${ }^{11,12}$. Los sangrados uterinos en usuarias de THM cíclica sugerimos que sean estudiados cuando sean prolongados (> 10 días), abundantes 0 intercíclicos frecuentes'.

En las usuarias de THM en esquema combinado continuo, administrándose el progestágeno todos los días junto con una dosis fija de estrógeno, más del $80 \%$ no tendrán sangrado, aunque un $15 \%$ puede presentar sangrado uterino esporádico los primeros 6 meses de uso. Por lo tanto, el SUPM en usuarias de THM en esquema combinado continuo deberían ser estudiados en aquellas mujeres que presenten un sangrado prolongado no esperado, abundante o persistente, o intermitente, por más de 6 meses de iniciada la THM, en especial en posmenopáusicas con más de 1 año de amenorrea'.

Especial mención merecen las pacientes posmenopáusicas con cáncer de mama y en tratamiento con tamoxifeno, en las que el riesgo de adenocarcinoma de endometrio aumenta de 3 a 6 veces en comparación con la población general. Este riesgo es proporcional a la dosis y el tiempo de uso del medicamento ${ }^{5}$. Sin embargo, cabe destacar que en las mujeres usuarias de tamoxifeno no se recomienda el tamizaje mediante ecografía, dada la alta tasa de falsos positivos en relación con los cambios endometriales observados con el uso de este fármaco ${ }^{13,14}$. En la actualidad, se sugiere estudiar solo a aquellas pacientes que presenten sangrado uterino, haciéndolo directamente a través de una biopsia endometrial ${ }^{14,15}$. Si la biopsia endometrial es fallida o la muestra es insuficiente para el diagnóstico, está indicado realizar un estudio de la cavidad endometrial para la búsqueda de lesiones focales, siendo la histeroscopía y la histerosonografía los procedimientos indicados. De estos, la 
histeroscopía presenta la ventaja de permitir la toma de biopsia o la resección de lesiones endometriales`.

\section{Exámenes propuestos a realizar en pacientes con sangrado uterino posmenopáusico}

\section{Ecografía transvagiinal}

La ecografía transvaginal es el método de elección para iniciar el estudio en toda paciente que presente un SUPM. Se indica a toda mujer posmenopáusica que manifieste sangrado uterino espontáneo y a mujeres posmenopáusicas usuarias de THM que presenten sangrado uterino no acorde con el esquema de THM utilizado. Debe llevarse a cabo antes de realizar la biopsia endometrial ${ }^{5}$.

Se ha definido un endometrio engrosado $\geq 4$ a 5 $\mathrm{mm}$ en mujeres posmenopáusicas con SUPM, con una sensibilidad para el diagnóstico de cáncer de endometrio del $95 \%$ y $96 \%$ respectivamente, y una especificidad del $46 \%$ y $51 \%{ }^{16}$. El American College of Obstetrics and Gynecology sugiere usar como medida máxima normal $<4 \mathrm{~mm}$ en pacientes con SUPM ${ }^{17}$. Esta medida límite no debe modificarse en pacientes usuarias de tamoxifeno o THM con SUPM, aun teniendo en cuenta que los falsos positivos pueden ser un poco superiores'.

En mujeres sin SUPM, el grosor endometrial es menos predictivo de una neoplasia endometrial. Las mujeres posmenopáusicas asintomáticas y con grosor endometrial $>11 \mathrm{~mm}$ tienen un riesgo de cáncer endometrial del 6,7\%; porcentaje similar al de las mujeres con SUPM y grosor endometrial $>5 \mathrm{~mm}$. Basado en esto, se sugiere realizar una toma de biopsia endometrial a toda paciente posmenopáusica sin sangrado con hallazgo de un grosor endometrial $>11 \mathrm{~mm}^{18}$. El uso de la ecografía transvaginal tiene la ventaja de ser poco invasivo, bien tolerado y de bajo costo. Por otra parte, su principal desventaja es la dificultad para discriminar entre patología benigna y maligna. Sin embargo, el uso del Doppler color permite evaluar el patrón vascular de las lesiones endometriales, aumentado la sospecha de patología maligna en presencia de vascularización aumentada y de baja resistencia ${ }^{16}$.

La medición del volumen endometrial y los índices de flujo Doppler medido por ecografía 3D no han demostrado ser superiores en la discriminación entre patología endometrial benigna o maligna, en comparación con la medición del grosor endometrial obtenida mediante ecografía transvaginal $2 \mathrm{D}^{16}$.

\section{Histerosonografía}

Recomendamos realizar este procedimiento en pacientes con SUPM y sospecha de lesión focal intracavitaria, siempre que no exista la posibilidad de contar con histeroscopía. Su principal desventaja es que no permite la toma de muestras de tejido endometrial para estudio histológico (no discrimina entre patología benigna o maligna) ${ }^{19}$. Puede no ser bien tolerada por la paciente, resultando hasta en un $15 \%$ de procedimientos fallidos. Aunque es un método de bajo costo, no reemplaza a la histeroscopía ${ }^{20}$.

\section{Biopsia endometrial}

Recomendamos realizarla idealmente mediante técnica aspirativa en forma ambulatoria, mediante el uso de cánula Pipelle ${ }^{\circledR}$ (dispositivo de plástico flexible y delgado que permite aspirar una muestra de tejido endometrial).

La sensibilidad de la biopsia para el diagnóstico de cáncer endometrial es mayor que la obtenida mediante la dilatación y curetaje $(99 \%$ vs. $92 \%$, respectivamente) ${ }^{21}$. Por tanto, se recomienda esta modalidad de estudio en primera instancia, ya que es más costo-efectiva. Cabe destacar que se estima que el $5 \%$ de las biopsias por Pipell ${ }^{\circledR}$ pueden ser reportadas como muestra insuficiente para el diagnóstico ${ }^{22}$; de ocurrir esta situación, un seguimiento ecográfico que informe de endometrio fino en una paciente asintomática permite no requerir mayor evaluación ${ }^{17}$.

En la tabla 1 se detallan las indicaciones actuales de biopsia endometrial aspirativa.

\section{Histeroscopía}

Recomendamos el uso de esta técnica cuando se sospeche una lesión focal o en pacientes en quienes no pueda realizarse una biopsia con Pipelle ${ }^{\circledR}$.

La ventaja de este procedimiento es que proporciona una visualización directa de la cavidad uterina, y además permite la toma de biopsia de áreas seleccionas o la extirpación de lesiones identificadas durante el procedimiento. Su sensibilidad para el diagnóstico de cáncer endometrial es del $98 \%$ y su especificidad es del $95 \%$. Entre sus desventajas están que requiere instrumental especial y entrenamiento adecuado del operador para realizar el procedimiento.

En la tabla 2 se resumen las principales indicaciones para la realización de una histeroscopía. 


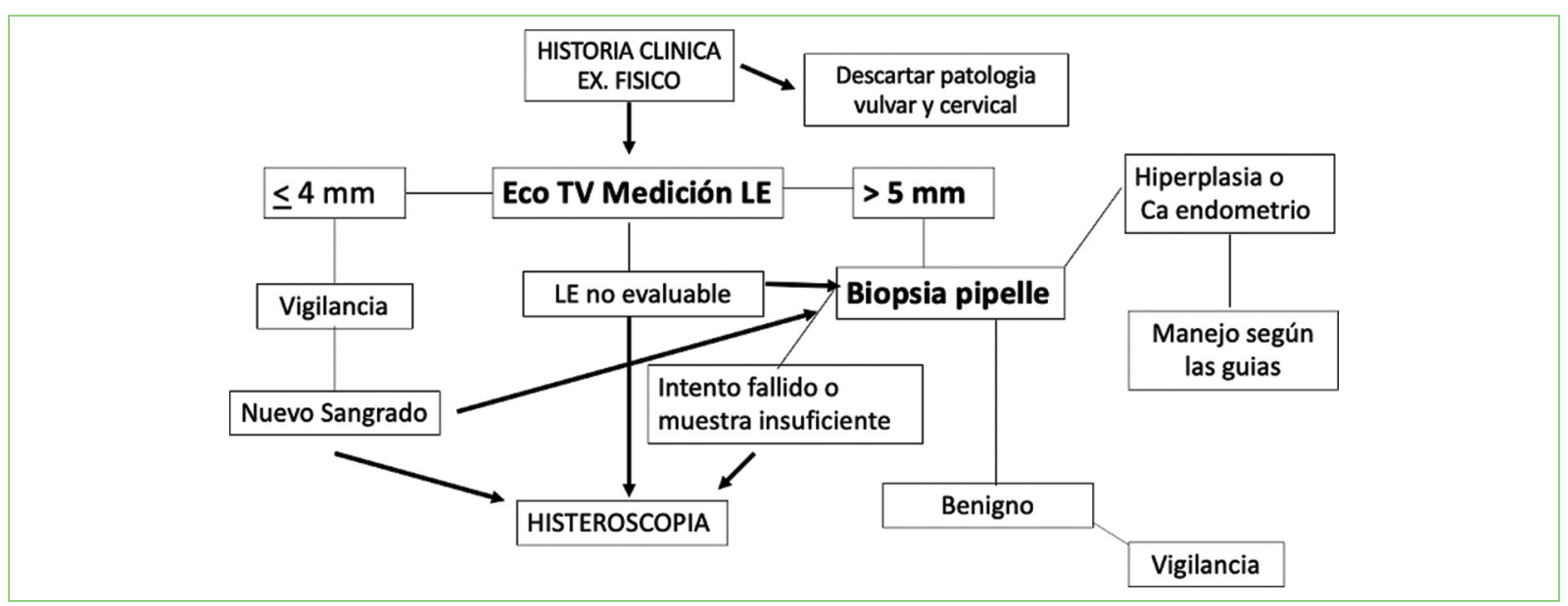

Figura 1. Flujograma diagnostico para la evaluación de las pacientes con sangrado uterino posmenopausico.

Tabla 1. Indicaciones de biopsia endometrial aspirativa

SUPM con grosor endometrial > $5 \mathrm{~mm}$ y $\sin$ THM

SUPM con grosor endometrial no evaluable en la ecografía transvaginal

SUPM persistente, independientemente del grosor endometrial

Sangrado atípico o endometrio $>11 \mathrm{~mm}$ en usuaria de THM

Usuaria de tamoxifeno con sangrado, independientemente del grosor endometrial

En pacientes con colección líquida intracavitaria y medición del grosor endometrial en bicapa $>3 \mathrm{~mm}$

SUPM: sangrado uterino posmenopáusico; THM: terapia hormonal de la menopausia.

Tabla 2. Indicaciones de histeroscopía oficinal

Sospecha de lesión focal: pólipo o mioma submucoso en ecografía transvaginal

Inadecuada visualización del endometrio por ecografía o fracaso de la histerosonografía

Pipelle ${ }^{\circledR}$ fallido (por cuello infranqueable o muestra insuficiente)

SUPM recurrente (definido como 2 episodios de sangrado con 2 biopsias por Pipelle ${ }^{\circledR}$ benignas tomadas con más de 6 meses de diferencia, en los últimos 2 años)

SUPM: sangrado uterino posmenopáusico.

Finalmente, en la figura 1 se propone un flujograma para el estudio de las pacientes que presentan SUPM.

\section{Conclusiones}

La evaluación de la paciente con sangrado uterino posmenopáusico debe iniciarse con un detallado interrogatorio dirigido y una exploración ginecológica exhaustiva que permita descartar otras causas de sangrado genital. El estudio comenzará con una ecografía transvaginal para definir la necesidad de realizar otras evaluaciones diagnósticas, como biopsia endometrial aspirativa para confirmar o descartar un cáncer de endometrio. La histeroscopía es una alternativa para la evaluación directa de la cavidad uterina en pacientes con estudios previos insatisfactorios o con sospecha de lesiones focales, permitiendo la toma de biopsias dirigidas de estas lesiones o su extirpación cuando sea factible, además del estudio histológico del endometrio.

\section{Financiamiento}

Este artículo no cuenta con financiamiento.

\section{Conflicto de intereses}

Los autores declaran no tener ningún conflicto de intereses.

\section{Responsabilidades éticas}

Protección de personas y animales. Los autores declaran que para esta investigación no se han realizado experimentos en seres humanos ni en animales. 
Confidencialidad de los datos. Los autores declaran que han seguido los protocolos de su centro de trabajo sobre la publicación de datos de pacientes.

Derecho a la privacidad y consentimiento informado. Los autores han obtenido el consentimiento informado de los pacientes y/o sujetos referidos en el artículo. Este documento obra en poder del autor de correspondencia.

\section{Bibliografía}

1. Munro M. Investigation of women with postmenopausal uterine bleeding: clinical practice recommendation. Perm J. 2014;18:55-70.

2. Ettinger B, Li DK, Klein R. Unexpected vaginal bleeding and associated gynecologic care in postmenopausal women using hormone replacement therapy: comparison of cyclic versus continuous combined schedules. Fertil Steril. 1998;69:865-9.

3. Astrup K, Olivarius N de F. Frequency of spontaneously occurring postmenopausal bleeding in the general population. Acta Obstet Gynecol Scand. 2004;83:203-7.

4. Mirkin S, Archer DF, Taylor HS, Pickar JH, Komm BS. Differential effects of menopausal therapies on the endometrium. Menopausia. 2014;21:899-908.

5. Braun MM, Overbeek-Wager EA, Grumbo RJ. Diagnosis and management of endometrial cancer. Am Fam Physician. 2016;93:468-74.

6. Burbos N, Musonda P, Giarenis I, Shiner AM, Giamougiannis P, Morris $\mathrm{E}$, et al. Age-related differential diagnosis of vaginal bleeding in postmenopausal women: a series of 3047 symptomatic postmenopausal women. Menopause Int. 2010;16:5-8.

7. Prendergast EN, Misch E, Chou YA, Roston A, Patel A. Insufficient endometrial biopsy results in women with abnormal uterine bleeding. Obstet Gynecol. 2014;23:180-1S.

8. Van Hanegem N, Breijer MC, Khan KS, Burger MPM, Mol BWJ, Timmermans A, et al. Diagnostic evaluation of the endometrium in postmenopausal bleeding: an evidence-based approach. Maturitas. 2011;68:155-64.

9. Clarke MA, Long BJ, Del Mar Morillo A, Arbyn M, Bakkum-Gamez JN Wentzensen N. Association of endometrial cancer risk with postmenopausal bleeding in women: a systematic review and meta-analysis. JAMA Intern Med. 2018;178:1210-22.
10. Nordal RR, Thoresen SO. Uterine sarcomas in Norway 1956-1992: incidence, survival and mortality. Eur J Cancer. 1997;33:907-11.

11. The NAMS 2017 Hormone Therapy Position Statement Advisory Panel. The 2017 hormone therapy position statement of The North American Menopause Society. Menopause. 2017;24:728-53.

12. Whitehead MI, Townsend PT, Pryse-Davies J, Ryder T, Lane G, Siddle NC, et al. Effects of various types and dosages of progestogens on the postmenopausal endometrium. J Reprod Med. 1982;27:539-48.

13. Gerber B, Krause A, Müller H, Reimer T, Külz T, Makovitzky J, et al. Effects of adjuvant tamoxifen on the endometrium in postmenopausal women with breast cancer: a prospective long-term study using transvaginal ultrasound. J Clin Oncol. 2000;18:3464-70.

14. Committee on Practice Bulletins-Gynecology. ACOG Practice Bulletin No. 126: Management of gynecologic issues in women with breast cancer. Obstet Gynecol. 2012;119:666-82.

15. Abdaal A, Mushtaq Y, Khasati L, Moneim J, Khan F, Ahmed H, et al. Post-menopausal bleeding - is transvaginal ultrasound a useful first-line investigation in tamoxifen users? Post Reprod Health. 2018;24:72-8

16. Long B, Clarke MA, Morillo ADM, Wentzensen N, Bakkum-Gamez JN. Ultrasound detection of endometrial cancer in women with postmenopausal bleeding: Systematic review and meta-analysis. Gynecol Oncol. 2020;157:624-33.

17. The role of transvaginal ultrasonography in the evaluation of postmenopausal bleeding. ACOG Committee Opinion No. 734. American College of Obstetricians and Gynecologist. Obstet Gynecol. 2018;131:e124-9.

18. Smith-Bindman R, Weiss E, Feldstein V. How thick is too thick? When endometrial thickness should prompt biopsy in postmenopausal women without vaginal bleeding. Ultrasound Obstet Gynecol. 2004;24:558-65.

19. Epstein E, Ramirez A, Skoog L, Valentin L. Transvaginal sonography, saline contrast sonohysterography, and hysteroscopy for the investigation of women with postmenopausal bleeding and endometrium $>5 \mathrm{~mm}$. UItrasound Obstet Gynecol. 2001;18:157-62.

20. Kaveh M, Sadegi K, Salarzaei M, Parooei F. Comparison of diagnostic accuracy of saline infusion sonohysterography, transvaginal sonography, and hysteroscopy in evaluating the endometrial polyps in women with abnormal uterine bleeding: a systematic review and meta-analysis. Wideochir Inne Tech Maloinwazyjne. 2020;15:403-15.

21. Kamel HS, Darwish AM, Mohamed SA. Comparison of transvaginal ultrasonography and vaginal sonohysterography in the detection of endometrial polyps. Acta Obstet Gynecol Scand. 2000;79:60-4.

22. Dijkhuizen FP, Mol BW, Brölmann HA, Heintz AP. The accuracy of endometrial sampling in the diagnosis of patients with endometrial carcinoma and hyperplasia: a meta-analysis. Cancer. 2000;89:1765-72. 